\title{
Fever with incontinence in the elderly: an approach for emergency medicine
}

\author{
Anthony Chow (Meds 2016) and Elaine Tang (Meds 2015) \\ Faculty Reviewer: Dr. Derrick Pringle, MD, FRCPC (Division of Emergency Medicine)
}

$\Lambda$ 72-year-old man develops a high fever and is taken to the ER by his concerned wife and daughter. During the intake interview, the wife reports that the man is experiencing worsening incontinence and occasionally complains of dysuria. Past medical records indicate that the man is already being treated and followed for hypertension and early Alzheimer's disease, but the wife notes that it has become increasingly difficult to manage her husband's confusion over the past week.

An emergency physician receives the above information from the triage nurse. The nurse adds that the man and his family have been waiting for two hours and all three appear quite distressed. How could such a situation be managed?

\section{GERIATRICS AND DEMENTIA: CONSIDERATIONS FOR THE BEDSIDE}

The age of a patient carries important implications for patient assessment and treatment. The physiology of a healthy, older adult adapts to functioning with tissues that have deteriorated with age, achieving a state of compromised balance termed homeostenosis. ${ }^{1}$ Patients in this subpopulation thus have a reduced capacity to respond and adapt to the stresses brought on by acute illness and are more vulnerable to such insults.

This affects patient assessment, as it may be difficult to distinguish symptoms of underlying disease from those of acute illness. Determining the patient's medical history, current medication regime and social circumstances is also challenging in an emergency setting. ${ }^{2}$ Prospective treatments are limited in that they must not exacerbate or interact with existing conditions and treatments. Polypharmacy, in particular, disproportionately affects the elderly and contributes to increased adverse drug events, physician visits, emergency admissions and hospitalisations. ${ }^{3}$

All the above can be further complicated by dementia in the patient. The patient's history may need to be obtained from family members or caregivers present in the ER or staff at a care facility, potentially leading to mistakes in interpreting symptom severity or missing incipient problems entirely. Furthermore, the patient may be unwilling or unable to participate in diagnostic or interventional measures that become indicated through the course of care. ${ }^{4}$

Pain management is especially difficult and no less important for patients with dementia. Though not ideal, primary care physicians must take care to detect surrogate markers of pain, such as confusion, gesturing or posturing, through the physical examination. ${ }^{4}$

Returning to the case, it is determined upon examination that the patient does not have the capacity to make his own medical decisions when he is unable to give his own name. Fortunately, his wife and daughter are attentive and meticulous principal caregivers. On request, they pro- duce a list of medications the patient is taking currently (chlorthalidone and ramipril for hypertension, galantamine for dementia, cyclazine for nausea, loratadine for seasonal allergies) and confirm that this degree of confusion is highly atypical. Unexpected change in cognitive status is a hallmark of pain in dementia, so $1000 \mathrm{mg}$ acetaminophen is ordered, ${ }^{5}$ after establishing that the patient would have no known allergy or drug interactions. Vital signs are assessed and no abnormalities beyond high blood pressure $(145 / 90)$ and high oral temperature $\left(40.5^{\circ} \mathrm{C}\right)$ are found.

\section{SIGNS OF INFECTION IN THE ELDERLY}

Fever as a sign of infection can operate quite differently in older adults. Ageing-mediated impairment of thermoregulation means that fever can fail to occur in up to half of elderly persons, despite the existence of lifethreatening infection. Even normal body temperature can be lower than expected in the elderly and even lower in those with dementia, due to the same age-related changes. ${ }^{6}$

Given the above, the finding of fever in the patient is a definite cause for concern. An explanation for this sign is sought in the next prominent set of symptoms: worsening incontinence and dysuria.

\section{URINARY INCONTINENCE: DIFFERENTIAL DIAGNOSIS}

Age is the greatest determinant of urinary incontinence risk. Many factors that negatively affect normal micturition are more likely to occur with increasing age. With respect to transient incontinence, these include urinary tract infections (UTIs), genitourinary muscle pathology, acute illnesses that mandate immobilisation or catheterisation, fecal impaction, medications with autonomic effects or psychological expression of dependency or rebellion.?

Though females are much more likely to experience incontinence than males, several urological causes of incontinence are exclusive to males. For younger males, in particular, urethral and prostatic infection secondary to a sexually-transmitted disease is a frequent cause of incontinence. ${ }^{8}$ In older men, incontinence is more often related to prostate disease. Prostate surgery is the most common cause of stress urinary incontinence in men and prostate hypertrophy may cause overflow incontinence by compressing the bladder. ${ }^{9}$ More importantly, UTIs are much more common in older than younger men, with an incidence approaching that seen in women. ${ }^{8}$

In the emergency department, life-threatening causes such as cauda equina syndrome, spinal cord compression and paraspinal abscess must additionally be ruled out if suspected. A bacterial abscess could be consistent with typical infection-related observations, like fever, and can lead to both the other conditions by direct or vascular compression. An MRI is necessary for ruling out this possibility. ${ }^{10}$ If additional lower body motor or sensory symptoms are present, it is important to more definitively determine whether the cauda equina or spinal cord is af- 
fected. ${ }^{11}$

In the present case, the physical examination is continued with an awareness of possible urological disease. Palpation of the abdomen does not reveal a distended or swollen bladder, which would have suggested overflow incontinence. ${ }^{7}$

As fever, dysuria and incontinence together point convincingly toward a urologic problem, an abdominal CT scan is not indicated at this point. Instead, blood is drawn for routine chemistry. A urethral catheterisation is performed to obtain a sterile urine sample for urinalysis and urine culture. The patient is mildly uncooperative but accepting of oral medication, so oral sedatives are administered. ${ }^{12} \mathrm{~A}$ bedside ultrasound bladder scan is ordered to rule out urinary retention and the need to leave the catheter in situ. ${ }^{13}$

An hour later, several reports are forwarded from the laboratory. Blood urea nitrogen (BUN) and creatinine are both normal, indicating good kidney function, especially for the patient's age. ${ }^{14}$ The routine urinalysis report, on the other hand, shows bacteria and leukocytes in high numbers in the urine (bacteriuria and pyuria) and the nitrate test is positive. This and urine turbidity shift suspicion to urinary tract infection (UTI) as opposed to the more common urethritis. ${ }^{8}$ Armed with all of the above, the doctor returns to the bedside.

\section{URINARY TRACT INFECTIONS: DIAGNOSIS}

In the elderly, investigation for UTIs needs to be justified by a high degree of suspicion because urinary incontinence and dysuria alone are very common and non-specific, as discussed. In fact, conditions like pollakiuria (frequent urination) and nocturia (night urination) are estimated to be present in over $50 \%$ of individuals over 60 years of age. ${ }^{15}$ In this population, even individuals without infection often present with the classic triad of UTI diagnosis: frequency, urgency and dysuria. ${ }^{16}$

Diagnosis of UTI in an older adult, then, often depends on nonurological indications. These commonly include a general decrease in day-to-day function, loss of weight or appetite, decreased alertness or flank or abdominal tenderness. For patients with dementia, behavioural deterioration and confusion can be extremely important symptoms. ${ }^{16}$

For the current patient, fever, confusion, dysuria and incontinence create a strong suspicion of UTI, strengthened by the subsequent findings of bacteriuria and pyuria. Upon further questioning, the daughter additionally confirms that lately the patient's appetite has decreased noticeably.

\section{URINARY TRACT INFECTIONS: MANAGEMENT IN THE EL- DERLY}

Diagnosis and treatment of UTIs in the emergency department frequently occurs without formal bacteria culturing. ${ }^{17}$ Though it is possible for asymptomatic urologic bacteremia to resolve spontaneously in the elderly, serious complications like renal damage, pyelonephritis and sepsis may occur, so it is prudent to treat all UTIs in the elderly as complicated. ${ }^{16,17}$

For most cases, a 10- or 14-day course of the broad-spectrum antibiotic levofloxacin is indicated. Particularly for elderly men, a fluoroquinolone like levofloxacin has the additional benefit of high prostate gland penetration. Recurrence and relapse are commonly seen in the elderly, so proper follow-up must be arranged. ${ }^{16}$

Returning to the case, the patient is prescribed oral levofloxacin, 250 $\mathrm{mg}$ per day for 14 days and allowed to take acetaminophen as needed. Given the fever and neurological symptoms, however, the patient is considered at risk of developing sepsis, so it is decided with the wife and daughter's consent that it would be most prudent to admit the patient to the medicine service.
The next day, the patient's serum lactate remains unremarkable and repeat measurement of his vitals - particularly heart rate and $\mathrm{O}_{2}$ saturation - return normal findings. He is cooperative and much more aware of his situation, correctly providing his name, the approximate date and the fact that he was in a hospital. ${ }^{18}$ Satisfied with the above improvements, the physician discharges the patient and asks the wife and daughter to help fill and administer the remainder of the prescription. They are also instructed to bring the patient to see his family doctor in one week to ensure clearance of the microorganism, confirm relief of neurological symptoms and consider management of incontinence, if it becomes chronic. Referral to a urologist is deemed unnecessary unless further complications occur.

\section{REFERENCES}

1. Mattison M, Marcantonio, ER. Hospital management of older adults [Internet]. UpToDate; 2012 Jan 26 [updated 2012 Jan 26; cited 2012 Nov 24]. Available from: http://www.uptodate.com/contents/hospital-management-ofolder-adults .

2. Wilber ST, Gerson LW, Terrell KM, Carpenter CR, Shah MN, Heard K, Hwang U. Geriatric emergency medicine and the 2006 Institute of Medicine reports from the Committee on the Future of Emergency Care in the U.S. Health System. Acad Emerg Med. 2006 Dec;13(12),1345-51.

3. Hueberger R. Polypharmacy and food-drug interactions among older persons: A review. J Nutr Gerontol Geriatr. 2012;31(4),325-403.

4. Valeriani L. Management of demented patients in emergency department. Int J Alzheimers Dis. 2011;2011,article ID 840312.

5. Miner JR. Analgesia and anesthesia in the emergency department. Emergency Medicine \& Critical Care. 2008;4,61-4.

6. Garlington W, High K. Evaluation of infection in the older adult [Internet]. UpToDate; 2012 Oct 16 [updated 2012 Oct 16; cited 2012 Nov 25]. Available from http://www.uptodate.com/contents/evaluation-of-infection-in-theolder-adult

7. Ouslander JG. Urinary incontinence in the elderly. West $\mathrm{J}$ Med. 1981;135(6),482-91.

8. Brusch JL. Urinary tract infections in males [Internet]. Medscape; $2012 \mathrm{Feb}$ 21 [updated 2012 Feb 21; cited 2012 Nov 24]. Available from emedicine. medscape.com/article/231574-overview .

9. Clemens JQ. Urinary incontinence in men [Internet]. UpToDate; 2012 Feb 7 [updated 2012 Feb 7; cited 2012 Nov 24]. Available from: http://www.uptodate.com/contents/urinary-incontinence-in-men .

10. Huff JS. Spinal Epidural Abscess [Internet]. Medscape; 2012 May 7 [updated 2012 May 7; cited 2012 Nov 24]. Available from: emedicine.medscape.com/ article/1165840-overview .

11. Dawodu ST. Cauda equina and conus medullaris syndromes [Internet]. Medscape; 2011 Aug 24 [updated 2011 Aug 24; cited 2012 Nov 24]. Available from: emedicine.medscape.com/article/1148960-overview .

12. Nassisi D, Okuda Y. ED Management of Delirium and Agitation. Emergency Medicine Practice. 2007;9(1).

13. Schaeffer AJ. Placement and management of urinary bladder catheters [Internet]. UpToDate; 2010 Oct 7 [updated 2010 Oct 7; cited 2012 Dec 20]. Available from: http://www.uptodate.com/contents/placement-and-managementof-urinary-bladder-catheters .

14. Vasavada SP. Urinary incontinence [Internet]. Medscape; 2012 Apr 5 [updated 2012 Apr 5; cited 2012 Nov 25]. Available from: emedicine.medscape. com/article/452289-overview .

15. Goepel M, Kirschner-Hermanns R, Welz-Barth A, Steinwachs KC, Rübben $H$. Urinary incontinence in the elderly: Part 3 of a series on incontinence. Dtsch Arztebl Int. 2010;107(30),531-6.

16. Murphy DP. Urinary tract infections in elderly patients: How best to diagnose and treat. Consultant. 2005;45(12). 


\section{THINKING ON YOUR FEET}

17. Foxman B. Epidemiology of urinary tract infections: Incidence, morbidity, and economic costs. Am J Med. 2002;113 Suppl 1A:5S-13S.

18. Bakker J, Jansen TC. Don't take vitals, take a lactate. Intensive Care Med. 2007;33(11),1863-5.
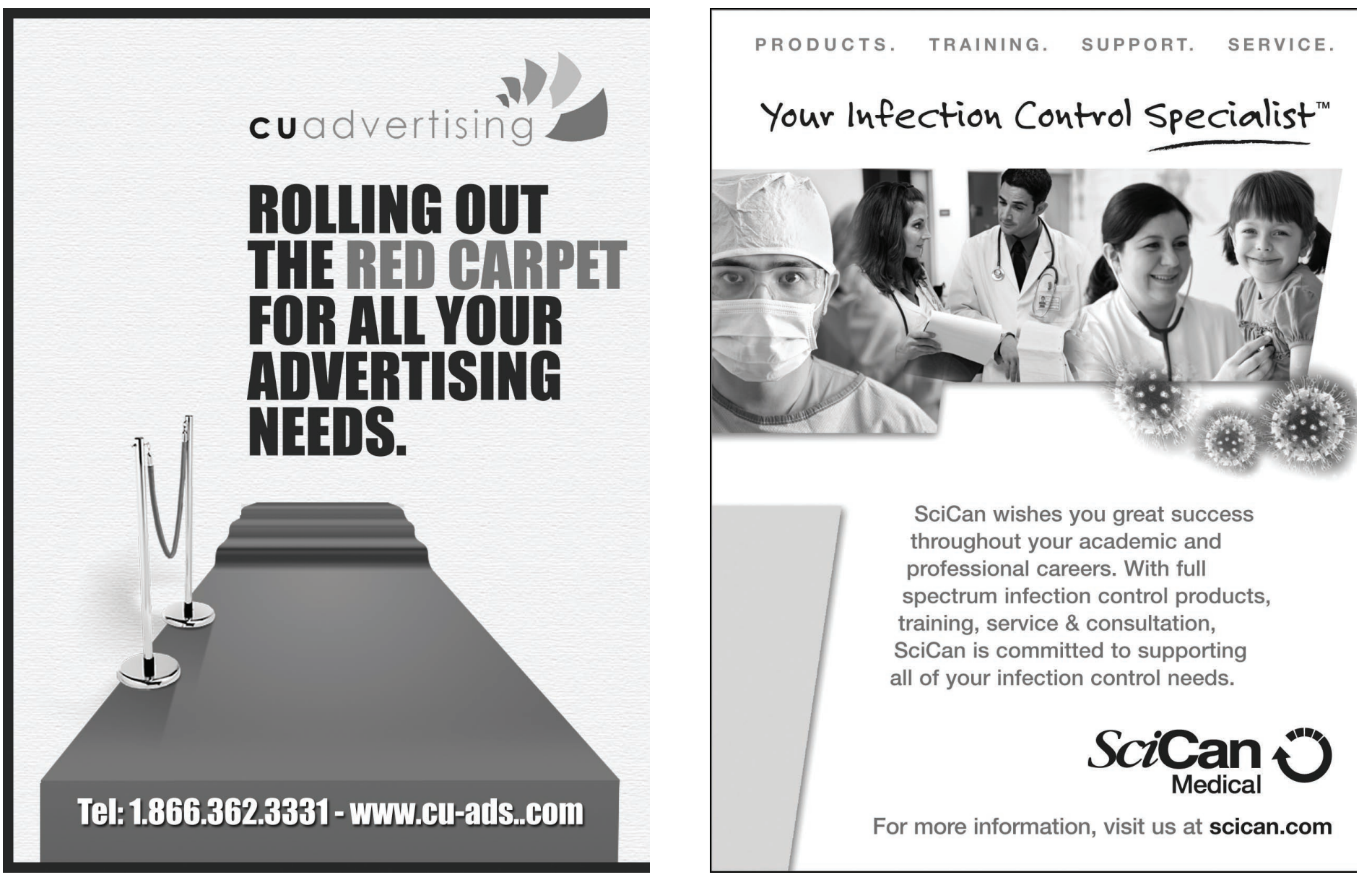